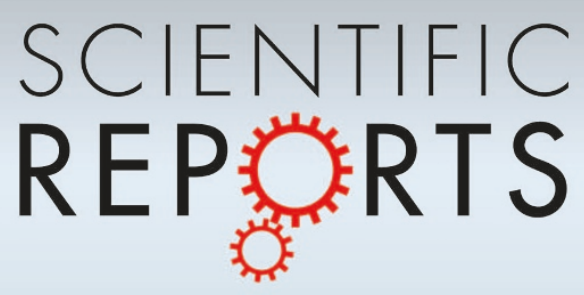

OPEN

SUBJECT AREAS:

RISK FACTORS

REPRODUCTIVE DISORDERS

NUTRITIONAL SUPPLEMENTS

Received

16 May 2014

Accepted

5 December 2014

Published

8 January 2015

Correspondence and requests for materials should be addressed to Z.-T.Z. (ztzhao@sdu.edu.

cn)

* These authors contributed equally to this work.

\section{Nausea and Vomiting in Early Pregnancy and the Risk of Neural Tube Defects: a Case-Control Study}

\author{
Qing-Bin Lu'*, Zhi-Ping Wang ${ }^{2 *}$, Li-Jie Gao ${ }^{2}$, Rui Gong ${ }^{2}$, Xi-Hong Sun ${ }^{2}$, Meng Wang ${ }^{2}$ \\ \& Zhong-Tang Zhao ${ }^{2}$
}

'School of Public Health, Peking University, Beijing, 100191, P. R. China, ${ }^{2}$ Department of Epidemiology and Health Statistics,
School of Public Health, Shandong University, Jinan, 250012, P. R. China.

There has been considerable professional debate on the association between nausea and vomiting in early pregnancy (NVP) and neural tube defects (NTDs) risk. This study explored the association between NVP and NTDs risk, and the effect of folic acid supplements on the association. A $1: 1$ matched case-control study was conducted and conditional logistic regression model was used to analyze the associations. The result showed the odds ratio (OR) of severe NVP for NTDs was 2.403 (95\%CI 1.437,4.017; $P<0.001)$ and that of moderate NVP was $1.469(95 \% \mathrm{CI} 1.063,2.031 ; P=0.020)$ compared with light NVP when adjusted by the potential confounders. Stratified by intake of folic acid supplements, the ORs for severe and moderate NVP turned to $2.147(95 \% \mathrm{CI} 1.140,4.043 ; P=0.018)$ and $2.055(95 \% \mathrm{CI} 1.320,3.199 ; P=0.001)$ in the stratum of non-intake of folic acid supplements while ORs reduced to $1.851(95 \% \mathrm{CI} 0.729,4.699 ; \mathrm{P}=0.195)$ and 1.003 $(95 \% \mathrm{CI} 0.594,1.694 ; \mathrm{P}=0.992)$ in the stratum of intake of folic acid supplements, respectively. We conclude that severe/moderate NVP has an association with the risk of NTDs, which was not found in the group with intake of folic acid supplements. Folic acid supplements should be recommended to use for the prevention of NTDs.

N eural tube defects (NTDs) are one group of severe defects in the central nervous system, always leading to infant deaths or disabilities. Data from the National Center for Birth Defects Monitor of China showed that the incidence rate of NTDs was 4.50 per ten thousand in the year of 2011. Lots of environmental and genetic factors have been proved to be risk factor of NTDs, such as smoking, passive smoking, obesity, lack of folic acid, methylenetetrahydrofolate reductase C677T mutation and so on ${ }^{1-12}$. Yet, there has been less professional debate on the association between nausea and vomiting in early pregnancy (NVP) and the risk of NTDs.

NVP is a common experience in pregnancy and the meta-analytic average rate was $69.4 \%$, but reported rates vary widely ${ }^{13}$. Meanwhile, NVP is found more often in Western countries and urban populations and rarely among Africans, Native Americans, Eskimos, and most Asian populations ${ }^{14}$. Although most women with NVP have symptoms limited to the first trimester from six weeks to 12 weeks, a small proportion of women have a prolonged course with symptoms extending until delivery. Women with severe NVP may suffer from hyperemesis gravidarum, an entity distinct from NVP, which may lead to maternal and fetal morbidity if left untreated.

Most studies have demonstrated that NVP is associated with a favorable outcome of the fetus. Weigel et al. found a strong significant association between NVP and decreased risk of miscarriage, but no consistent associations with perinatal mortality ${ }^{15}$. However, an increased risk of intrauterine growth retardation and low birth weight was associated with the women with severe $\mathrm{NVP}^{16,17}$. Previous studies also showed no difference in congenital abnormalities between those with and without $\mathrm{NVP}^{11}$ and a lower risk of congenital heart defects in infants born to women with early onset of NVP requiring antiemetic use compared to women without nausea ${ }^{18}$.

However, there has been considerable professional debate on the association between NVP and the risk of NTDs. Czeizel et al. suggested an inverse association between severe NVP and the risk of some congenital abnormalities, including NTDs ${ }^{19}$. While this association was not observed in other studies ${ }^{20,21}$. NVP may reduce energy intake and levels of anabolic hormones, insulin, and insulin growth factor leading to a shunting of scarce nutrients to the placenta and fetus ${ }^{22}$, also including maternal level of folic acid. The evidence of public health suggests that daily folic acid supplements have a significant protective effect in preventing NTDs. It remains unknow whether the folic acid supplements intake would affect the association between NVP and NTDs. 
Table 1 | Characteristics of the subject women in the case and control groups

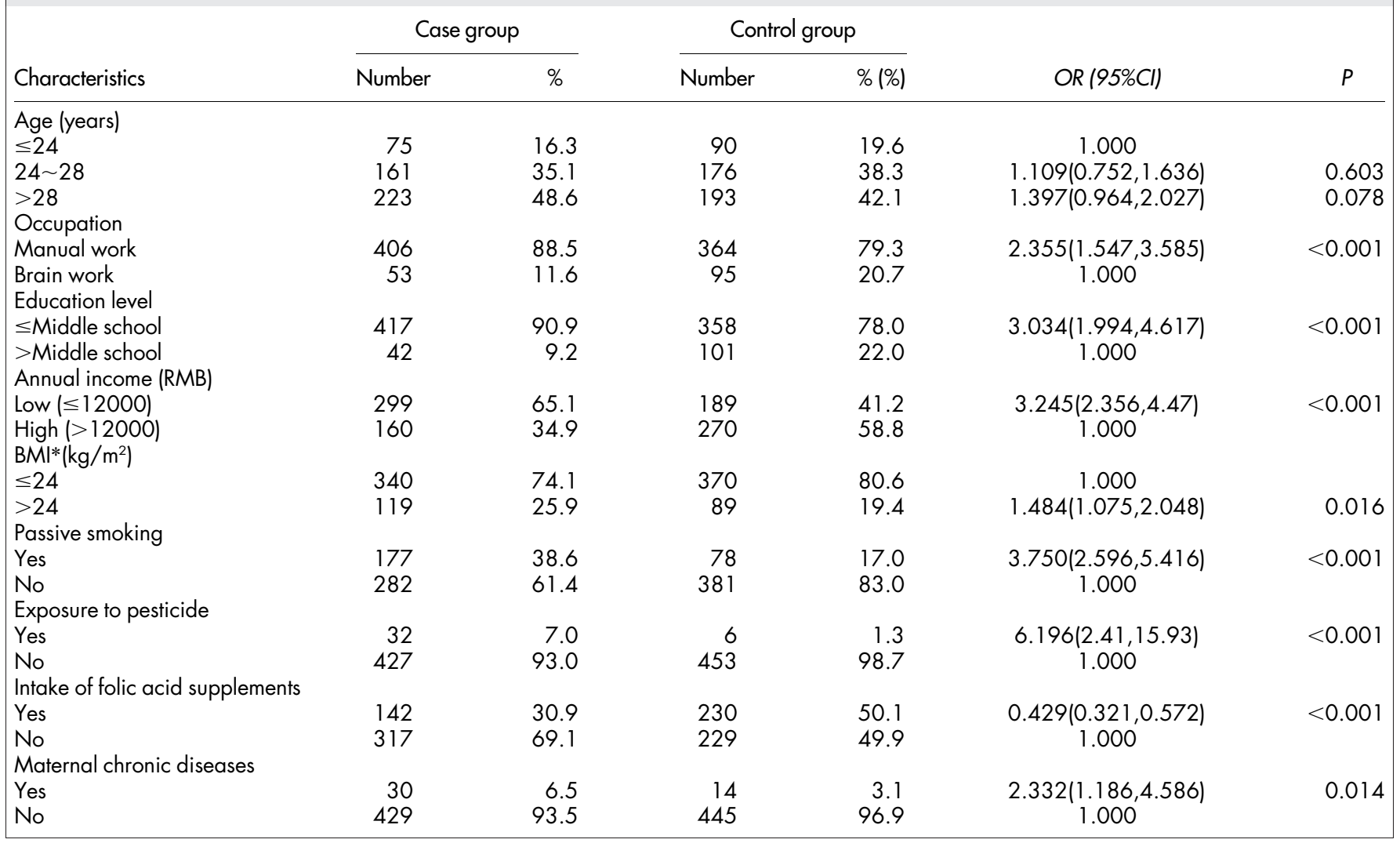

Meanwhile, the severity of nausea and vomiting was associated with the frequency of feelings of depression and adverse effects on women's relationships, which may have an effect on the risk of $\mathrm{NTDs}^{23}$.

Therefore, we conducted a matched case-control study in China to further explore the association between NVP and NTDs risk, and the effect of folic acid supplements on the association between NVP and NTDs risk.

\section{Methods}

The 1:1 matched case-control study was conducted in Shanxi Province and Shandong Province of China. We identified study subjects and collected the information from the registration cards and hospital records through the National Center for Birth Defects Monitor of China, the Maternal and Child Care Service Centers, the Family Planning Centers and the local hospitals. We excluded those without detailed home address and contact information.

Cases were the women with any infant or fetus with a diagnosis (ICD-10) of anencephaly (Q00.0), spina bifida (Q05), or encephalocele (Q01) at birth or prenatally within 2 years before the survey date (from March to December 2008). Controls were the women with live born infants, randomly selected without an apparent congenital malformation. The matching criteria were that the matched case and control were from the same region, the same hospital and childbirth in the same year in order to control the potential confounding factors by the above factors and reduce the sample size.

In the study, structured questionnaires were used for data collection. The questionnaire contained questions on maternal socio-demographic characteristics (age, education, occupation, and annual income), conditions of fetuses or infants, height and prepregnancy weight, exposure to passive smoking and pesticide, chronic diseases (anemia, diabetes, epilepsy, hyperthyroidism, tumor and hypertension), fever or cold, NVP status, intake of folic acid supplements and nutritional status in the periconceptional period. The questionnaires were administered through householdto-household survey by well-trained interviewers who had no knowledge of the main hypotheses of the study. The study protocol was reviewed and approved by the Ethics Review Committee of Public Health at Shandong University. The methods were carried out in accordance with the approved guidelines. Informed consent forms were shown to the subjects and their spouses, and signed by the subjects with the assistance of community/village doctors.

Severity of NTD was categorized by three stages: light NVP means one or none of most situation in early pregnancy with salivation or mild nausea and vomiting in early morning, which would not affect daily life; moderate NVP means that the symptoms of nausea and vomiting aggravate and occur to be not limited to the morning, which can be released after an adequate rest; severe NVP means the continuous symptoms of nausea and vomiting occur and the women need to rest in bed or be hospitalized.

Descriptive statistics were calculated for all variables; continuous variables were summarized as means and standard deviations or median and range, and categorical variables were summarized as frequencies and proportions. To determine the difference between groups, independent $t$ test, chi-square test, or Fisher exact was used where appropriate. Body mass index (BMI) was calculated by prepregnancy weight $(\mathrm{kg})$ divided by the square of height $(\mathrm{m})$. Differences of categorical data between the case women and the control women were examined by conditional logistic regression models which were used to calculate odds ratios (ORs) and their 95\% confidence intervals (CIs). Conditional logistic regression model was used in order to analyze the association between NVP and NTDs stratified by the variable of intake of folic acid supplements. Adjusted ORs and their $95 \%$ CIs were calculated by adjusting for the potential confounders. A two-sided P value of less than 0.05 was considered to be statistically significant. All analyses were performed using SAS software, version 9.1.3 (SAS Institute, Cary, NC, USA).

\section{Results}

Totally 459 case women and 459 control women were recruited in the study. There were 194 anencephaly, 200 spina bifida and 65 encephalocele in the case group. The characteristics of the women in the case and control groups were presented in Table 1. The differences between the case and the control groups were statistically significant for occupation, education level, annual income, BMI, passive smoking, exposure to pesticide, intake of folic acid supplements and maternal chronic diseases (all $\mathrm{P}<0.05$ ) except for age.

NVP distribution in case and control groups and the OR values between NVP and NTDs risk stratified by the variable of intake of folic acid supplements were shown in Table 2 . In the case women, $11.5 \%$ (53/459) had severe NVP and 29.0\% (133/459) had moderate NVP in the first trimester. In the control women, 6.1\% (28/459) had severe NVP and $24.6 \%(113 / 459)$ had moderate NVP in the first trimester. The OR of NTDs for the women with severe NVP was 2.403 (95\%CI 1.437,4.017; $P<0.001)$ and that with moderate NVP was $1.469(95 \%$ CI $1.063,2.031 ; P=0.020)$, compared to the women 
Table 2 | NVP distribution in case and control groups and the OR values between NVP and NTDs stratified by intake of folic acid supplements

\begin{tabular}{|c|c|c|c|c|c|c|c|}
\hline \multirow[b]{2}{*}{ Factor } & \multirow{2}{*}{$\frac{\text { Case }}{\mathrm{n}=459}$} & \multirow{2}{*}{$\frac{\text { Control }}{n=459}$} & \multirow[b]{2}{*}{$P$} & \multicolumn{2}{|l|}{ Crude } & \multicolumn{2}{|l|}{ Adjusted* } \\
\hline & & & & OR (95\%Cl) & $P$ & OR $(95 \% \mathrm{Cl})$ & $P$ \\
\hline \multicolumn{8}{|l|}{ NVP\# } \\
\hline Light & 273 & 318 & 0.002 & 1.000 & & 1.000 & \\
\hline Moderate & 133 & 113 & & $1.469(1.063,2.031)$ & 0.020 & $1.626(1.117,2.366)$ & 0.011 \\
\hline Severe & 53 & 28 & & $2.403(1.437,4.017)$ & $<0.001$ & $1.966(1.098,3.523)$ & 0.023 \\
\hline \multicolumn{8}{|l|}{ Intake of folic acid supplements" } \\
\hline Light NVP & 97 & 155 & 0.566 & 1.000 & & 1.000 & \\
\hline Moderate NVP & 35 & 64 & & $1.003(0.594,1.694)$ & 0.992 & $0.967(0.537,1.744)$ & 0.912 \\
\hline Severe NVP & 10 & 11 & & $1.851(0.729,4.699)$ & 0.195 & $1.920(0.710,5.197)$ & 0.199 \\
\hline \multicolumn{8}{|l|}{ Non-intake of folic acid supplements" } \\
\hline Light NVP & 176 & 163 & $<0.001$ & 1.000 & & 1.000 & \\
\hline Moderate NVP & 98 & 49 & & $2.055(1.320,3.199)$ & 0.001 & $2.292(1.408,3.730)$ & 0.001 \\
\hline Severe NVP & 43 & 17 & & $2.147(1.140,4.043)$ & 0.018 & $2.103(1.058,4.180)$ & 0.034 \\
\hline
\end{tabular}

with light NVP when adjusted by the potential confounders. The interaction between NVP and intake of folic acid supplements demonstrated a significantly antagonistic effect $(\mathrm{P}=0.034)$. Interestingly, when stratified by intake of folic acid supplements, the ORs for severe and moderate NVP turned to 2.147 (95\%CI $1.140,4.043 ; P=0.018)$ and $2.055(95 \% \mathrm{CI} 1.320,3.199 ; P=0.001)$ compared to light NVP in the stratum of non-intake of folic acid supplements, respectively. While in the stratum of intake of folic acid supplements, the ORs for severe and moderate NVP reduced to 1.851 $(95 \%$ CI $0.729,4.699)$ and $1.003(95 \%$ CI $0.594,1.694)$, respectively, which demonstrated no statistically significant differences compared with light $\operatorname{NVP}(\mathrm{P}=0.195$ and $\mathrm{P}=0.992)$.

The distribution and risk of NVP for the different subtypes of NTDs were shown in Table 3 and Table 4 . The risk of anencephaly was significantly associated with severe NVP and the OR value was $3.738(95 \% \mathrm{CI} 1.158,12.073 ; P=0.028)$ when adjusted by the potential confounders. However, no statistically significant associations were found between NVP and spina bifida/encephalocele (all $P>0.05$ ). When stratified by intake of folic acid supplements, the risk of severe and moderate NVP for anencephaly rose in the group of non-intake of folic acid supplements and reduced to no statistically significant differences in the group of intake of folic acid supplements. For spina bifida, it showed no differences stratified by intake of folic acid supplements.

\section{Discussion}

This study had explored the association between NVP and NTDs risk. The results showed that severe or moderate NVP had a significant association with the risk of NTDs. While this association was not found in the group with the intake of folic acid supplements.

The etiology of NVP has been well understood, but it is widely believed that physiological, psychological, genetic, and cultural components are contributing factors ${ }^{14,18}$. The occurrence of NVP may be of benefit in pregnancy and less likely to result in miscarriage, preterm delivery, or intrauterine growth retardation. The Maternal and Embryo Protection Hypothesis posits that pregnancy sickness results in adaptive dietary changes that reduce the exposure of the fetus and mother to dietary pathogens, toxins and parasites by reducing the intake of foods such as meat, fish and dairy products ${ }^{24}$. But the study by Weigel et al. was little direct evidence for this hypothesis ${ }^{25}$. Studies suggested that NVP was promoted by secretion of human chorionic gonadotrophin (hCG), largely on the basis of the coincidence between the onset of NVP and hCG secretion and the peaks of both at around 12 to 14 weeks in the first trimester ${ }^{26-28}$. Abnormal concentrations of hCG are associated with poor pregnancy outcomes. It seems that NVP and hCG are related and nutrient restriction during critical periods of embryonic development may ensure production of hCG at optimal levels to protect placental development and optimize nutrient partitioning between maternal and fetal tissues.

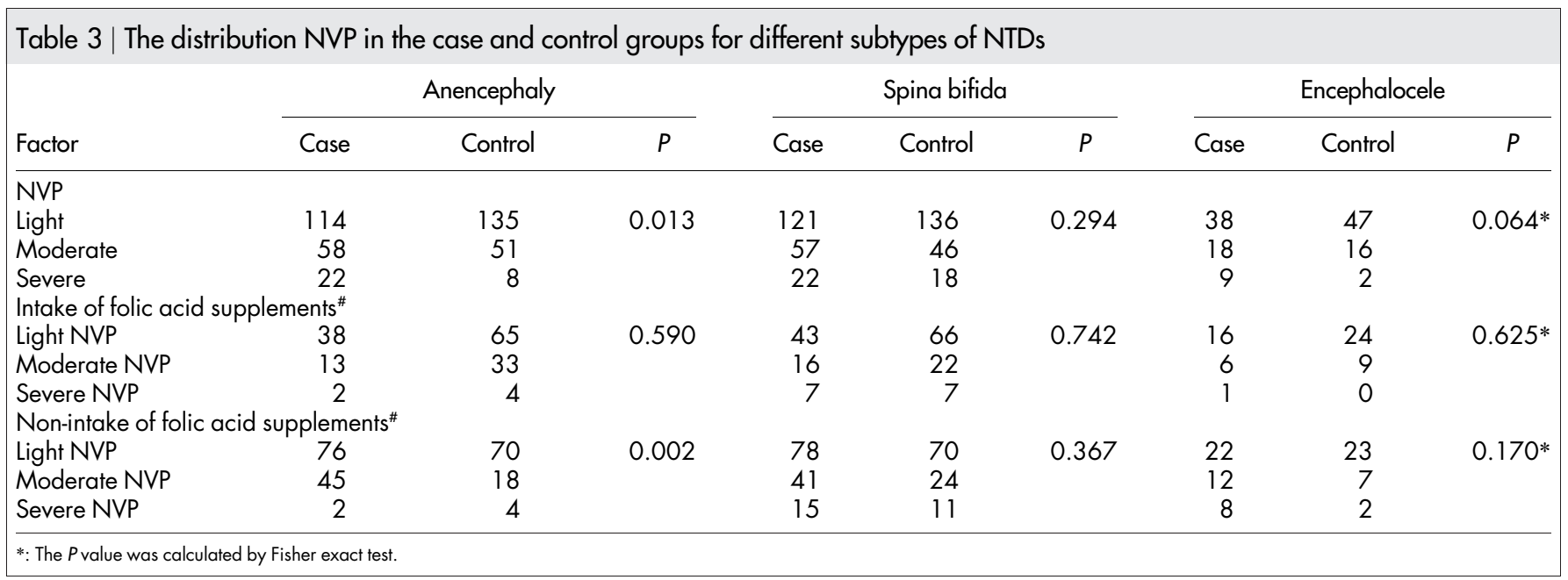


Table 4 | Adjusted OR values between NVP and different subtypes of NTDs stratified by intake of folic acid supplements

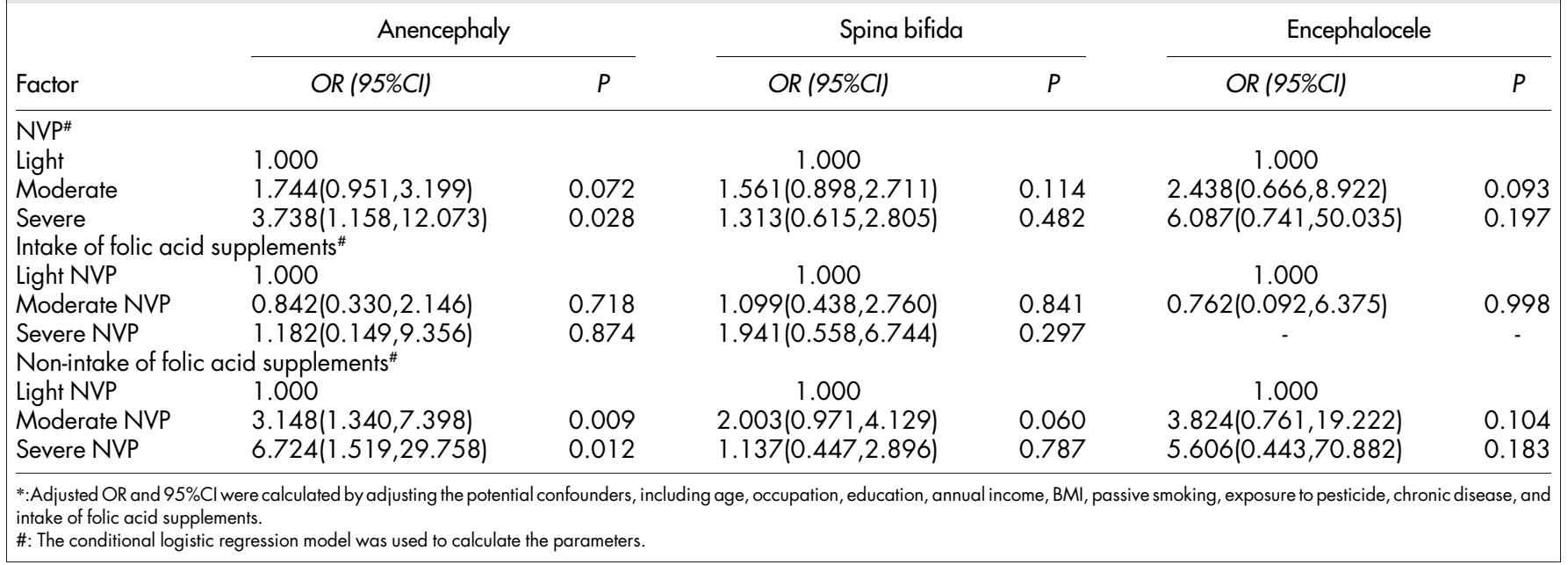

In our study, we found an opposite effect: a higher level of pregnancy sickness was correlated with a worse pregnancy outcome- a higher likelihood of neural tube defects. The advocates of the maternal and embryo protection hypothesis have not considered the potential costs of pregnancy sickness, in particular, reduced dietary intake. Lee et al. reported that morning sickness in pregnant women result in poor dietary diversity and reduced intakes of energy, protein, and micronutrients and, consequently, adversely affected infant outcome as well as maternal weight gain during early pregnancy ${ }^{29}$. In that case, reduced dietary intake certainly will influence or decrease the level of folic acid, which is mainly depending on food intake. It has been proved that folate deficiency could increase the risk of NTDs while intake of folic acid supplements could decrease the risk of NTDs ${ }^{11}$, which is also observed from our study. The result demonstrated that severe NVP was related to the risk of NTDs in this study. Stratified by the intake of folic acid supplements, the risk of NTD by severe NVP was decreased in the group with intake of folic acid supplements. The assumption is partly proved that severe NVP may reduce the intake of nutrition and then lower the level of folic acid in the maternal body, leading to the increased risk of NTDs. These results were different from those by Czeizel et $\mathrm{al}^{30}$. The effect of NVP on NTDs risk may depend on the level of hCG and folic acid. Of course, drug treatment on NVP was not considered as a limitation in this study.

In the study, the risk of anencephaly was associated with severe NVP, while spina bifida and encephalocele were not. The NVP may have different effects on the risk of anencephaly and the other two subtypes, which means, the hypothesis was further supported that anencephaly had a different pathogenic mechanism from the other two subtypes. Anencephaly may be more sensitive than spina bifida and encephalocele to folate deficiency. Folic acid supplements are likely to improve the status of folate level in the maternal body changed by NVP.

Although some confounders have been adjusted, considering other potential confounders, both NVP and NTDs might be the results of some underlying conditions. The association between NVP and NTDs needs further study considering more potential risk factors. The results showed a strong effect of severe NVP on the risk of encephalocele. However, the actual frequency in both of the case and control women used for calculation was too small to be convincing. Further studies are needed to clarify the actual relationship between severe NVP and the risk of encephalocele.

In conclusion, this case-control study reveals that severe or moderate NVP has an association with the risk of NTDs, which was not found in the group with intake of folic acid supplements. So folic acid supplements should be recommended within three months before pregnancy or in the first trimester to use for prevention of NTDs.

1. Gao, L. J., Zhao, Z. T., Li, D., Jiang, B. F. \& Hao, F. R. A case-control study on the risk factors of central nervous system congenital malformations. Chin J Epidemiol 25, 794-798 (2004)

2. Fang, W., Yan-fang, Y. \& Pei-zhen, L. A case-control study on the risk factors of neural tube defects in Shanxi province. Chin J Epidemiol 29, 771-774 (2008).

3. Rasmussen, S. A., Chu, S. Y., Kim, S. Y., Schmid, C. H. \& Lau, J. Maternal obesity and risk of neural tube defects: a metaanalysis. Am J Obstet Gynecol 198, 611-619 (2008).

4. Molloy, A. M. et al. Maternal vitamin B12 status and risk of neural tube defects in a population with high neural tube defect prevalence and no folic Acid fortification. Pediatrics 123, 917-923 (2009).

5. Stothard, K. J., Tennant, P. W., Bell, R. \& Rankin, J. Maternal overweight and obesity and the risk of congenital anomalies: a systematic review and metaanalysis. JAMA 301, 636-650 (2009).

6. Wolff, T., Witkop, C. T., Miller, T. \& Syed, S. B. Folic acid supplementation for the prevention of neural tube defects: an update of the evidence for the U.S. Preventive Services Task Force. Ann Intern Med 150, 632-639 (2009).

7. Yazdy, M. M., Liu, S., Mitchell, A. A. \& Werler, M. M. Maternal Dietary Glycemic Intake and the Risk of Neural Tube Defects. Am J Epidemiol 171, 407-414 (2009).

8. Aguilar-Garduno, C., Lacasana, M., Blanco-Munoz, J., Borja-Aburto, V. H. \& Garcia, A. M. Parental occupational exposure to organic solvents and anencephaly in Mexico. Occup Environ Med 67, 32-37 (2010).

9. Harisha, P. N., Devi, B. I., Christopher, R. \& Kruthika-Vinod, T. P. Impact of 5,10methylenetetrahydrofolate reductase gene polymorphism on neural tube defects. J Neurosurg Pediatr 6, 364-367 (2010).

10. Yin, Z. et al. A population-based case-control study of risk factors for neural tube defects in Shenyang, China. Childs Nerv Syst 27, 149-154 (2011).

11. Zur, E. Nausea and vomiting in pregnancy: a review of the pathology and compounding opportunities. Int J Pharm Compd 17, 113-123 (2013).

12. Wang, M., Wang, Z. P., Zhang, M. \& Zhao, Z. T. Maternal passive smoking during pregnancy and neural tube defects in offspring: a meta-analysis. Arch Gynecol Obstet 289, 513-521 (2014).

13. Einarson, T. R., Piwko, C. \& Koren, G. Quantifying the global rates of nausea and vomiting of pregnancy: a meta analysis. J Popul Ther Clin Pharmacol 20, e171-183 (2013).

14. Lee, N. M. \& Saha, S. Nausea and vomiting of pregnancy. Gastroenterol Clin North Am 40, 309-334 (2011).

15. Weigel, R. M. \& Weigel, M. M. Nausea and vomiting of early pregnancy and pregnancy outcome. A meta-analytical review. Br J Obstet Gynaecol 96, 1312-1318 (1989).

16. Deuchar, N. Nausea and vomiting in pregnancy: a review of the problem with particular regard to psychological and social aspects. BrJ Obstet Gynaecol 102, 6-8 (1995).

17. Zhou, Q., O'Brien, B. \& Relyea, J. Severity of nausea and vomiting during pregnancy: what does it predict? Birth 26, 108-114 (1999).

18. Boneva, R. S., Moore, C. A., Botto, L., Wong, L. Y. \& Erickson, J. D. Nausea during pregnancy and congenital heart defects: a population-based case-control study. Am J Epidemiol 149, 717-725 (1999). 
19. Czeizel, A. E., Puho, E., Acs, N. \& Banhidy, F. Inverse association between severe nausea and vomiting in pregnancy and some congenital abnormalities. Am J Med Genet A 140, 453-462 (2006).

20. Wu, Y.-Q. et al. A case-control study of neural tube defects in China. Chin J Med Gene 11, 210-213 (1994).

21. Anderka, M. et al. Medications used to treat nausea and vomiting of pregnancy and the risk of selected birth defects. Birth Defects Res A Clin Mol Teratol 94 22-30 (2012).

22. Czeizel, A. E., Dudas, I., Vereczkey, A. \& Banhidy, F. Folate deficiency and folic acid supplementation: the prevention of neural-tube defects and congenital heart defects. Nutrients 5, 4760-4775 (2013).

23. Mazzotta, P., Maltepe, C., Navioz, Y., Magee, L. A. \& Koren, G. Attitudes, management and consequences of nausea and vomiting of pregnancy in the United States and Canada. Int J Gynaecol Obstet 70, 359-365 (2000).

24. Flaxman, S. M. \& Sherman, P. W. Morning sickness: a mechanism for protecting mother and embryo. Q Rev Biol 75, 113-148 (2000).

25. Weigel, M. M. et al. Food aversions and cravings during early pregnancy: association with nausea and vomiting. Ecol Food Nutr 50, 197-214 (2011).

26. Boskovic, R., Rudic, N., Danieliewska-Nikiel, B., Navioz, Y. \& Koren, G. Is lack of morning sickness teratogenic? A prospective controlled study. Birth Defects Res A Clin Mol Teratol 70, 528-530 (2004).

27. Huxley, R. R. Nausea and vomiting in early pregnancy: its role in placental development. Obstet Gynecol 95, 779-782 (2000).

28. Forbes, S. Pregnancy sickness and embryo quality. Trends Ecol Evol 17, 115-120 (2002).

29. Lee, J.-I., Lee, J.-A. \& Lim, H.-S. Morning sickness reduces dietary diversity, nutrient intakes, and infant outcome of pregnant women. Nutr Res 24, 531-540 (2004).
30. Davis, M. Nausea and vomiting of pregnancy: an evidence-based review. J Perinat Neonatal Nurs 18, 312-328 (2004).

\section{Acknowledgments}

This work was supported by the project of the effectiveness evaluation of hospital-based comprehensive birth defects intervention methods, The Ministry of Science and Technology, China (2006BA105A01).

\section{Author contributions}

Conceived and designed the experiments: Z.T.Z. Performed the experiments: Q.B.L. Z.P.W. L.J.G. M.W. X.H.S. R.G. Analyzed the data: Q.B.L. Z.P.W. Z.T.Z. Contributed reagents/ materials/analysis tools: Q.B.L. Z.P.W. L.J.G. X.H.S. R.G. M.W. Wrote the paper: Q.B.L. Z.P.W. Z.T.Z.

\section{Additional information}

Competing financial interests: The authors declare no competing financial interests.

How to cite this article: Lu, Q.-B. et al. Nausea and Vomiting in Early Pregnancy and the Risk of Neural Tube Defects: a Case-Control Study. Sci. Rep. 5, 7674; DOI:10.1038/ srep07674 (2015)

(i) $\Theta$ This work is licensed under a Creative Commons Attribution-NonCommercialNoDerivs 4.0 International License. The images or other third party material in this article are included in the article's Creative Commons license, unless indicated otherwise in the credit line; if the material is not included under the Creative Commons license, users will need to obtain permission from the license holder in order to reproduce the material. To view a copy of this license, visit http:// creativecommons.org/licenses/by-nc-nd/4.0/ 